\title{
Thérèse à la lumière de Clara
}

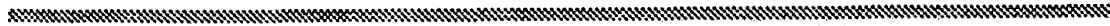

Magalie D. Hanquier

1

ombre de critiques ont cherché commentThérèse Desqueyroux illustre la pensée catholique de Mauriac. Quels rapports établit-t-il entre les pharisiens et la publicaine (Séailles 134) ?' Introduit-il la grâce dans son oeuvre? De telles questions peuvent sans doute conduire à une réflexion sur les rapports de l'écrivain et de sa religion, mais rendent-elles compte de la valeur littéraire de l'oeuvre? Nul besoin n'est de se situer dans une perspective catholique pour se sentir envoûter par ce "chant" selon le mot de Bernard Chochon (11). ${ }^{2}$ Si Thérèse Desqueyroux est comme le dit Pierre-Henri Simon "une réalité spirituelle exprimée par des voies poétiques" (Chochon 11), peutêtre nous faut-il explorer la structure métaphorique du roman. En observant attentivement comment s'organisent les ressemblances et les contrastes entre les personnages et de quelle manière Mauriac fixe et transmute autour d'eux quelques figures obsédantes, peut- être verra-t-on apparaître le fin réseau des rimes, des assonances et des rythmes du texte. Plutôt que la trop limpide anecdote, nous choisirons comme guide dans cette nébuleuse de significations le personnage le plus négligé des critiques : Clara. 3

Clara, tante et substitut matemel de Therèse, appartient au bloc familial et peut être comparế à la mère de Bernard dont elle présente les mêmes qualités de dévotion a l'enfant. En cela, elle semble une "femme de la famille" $(T, 86), 4$ même si sa surdité et sa vie retirêe à Argelouse l'empêchent de prendre réellement part aux intérêts familiaux. Cependant, Clara n'est qu'une mère adoptive. Vieille fille, c'est-à-dire jeune fulle, elle n'a jamais été “aimée ni possédée" (T, 105). Selon la philosophie de l'oeuvre, cela signifie qu'elle a été sevrée d'amour, mais essentiellement qu'elle a échappé a "cette ineffaçable salissure des noces" (T, 36) : elle est demeurcé dans la pureté de l'adolescence. Clara 
appartient donc, de ce premier point de vue, à deux mondes, celui des jeunes filles par sa pureté et celui des femmes de la famille par son rôle de mère. Pourtant, à la différence des jeunes filles, elle n'éprouve aucune passion amoureuse, comme Thérèse adolescente pour Anne, ou celle-ci pour Jean. Or, même la mère de Bernard semble avoir éprouvé l'amour durant son veuvage état proche de celui de jeune fille - seule explication possible de son mariage avec cet Hector de la Trave "sans le sou" ( $T$, 42). A travers le personnage de Clara, l'on voit donc s'élaborer des catégories d'appartenance auxquelles elle échappe toujours en partie mais qui permettent peut-être d'organiser un schéma global à l'intérieur duquel les personnages se définiront par leurs appariements et leurs oppositions.

Toute la société - représentée par le cocher, les valets, le docteur, le pharmacien, l'avocat, des dames dans la gare, les paysans en général - semble fonctionner comme un adjuvant de la famille en opposition à Therèse, ou si l'on veut comme le cadre étendu de leur conflit. Mais cette société ne constitue pas seulement un fond de toile indifférencié : des homologies apparaissent entre ses membres. Ainsi, l'avocat tout comme le docteur demeurent indifférents non seulement au sort de Thérèse, mais aussi à celui des familles Desqueyroux et Laroque. Qu'on ne fasse pas appel à une compétence supérieure suffit à leur satisfaction. Les cochers et les valets éprouvent le désir d'observer Thérèse. Tous les paysans manifestent le même mépris à son égard et cherchent à lobserver sans courir de risques. En introduisant des figurants qui se répondent d'une extrémité du livre à l'autre, Mauriac leur confere un caractère de généralité tout en réalisant une structure harmonieuse. Lorsque nous retrouvons dans un autre contexte les mêmes regards avides ou les mêmes attitudes abjectes, nous avons le sentiment que ces éléments infiltrent tout l'ouvrage, et l'éclairent d'une lumière sinistre qui conditionne toute notre perception.

Entre les membres de la famille, les traits communs sont aisément discernables, ils procèdent de l'hérédité et des rôles que dans cette société traditionnelle chacun est amené à jouer. La famille Laroque semble s'opposer à la famille Desqueyroux sur un plan politico-religieux, mais en réalité les mêmes valeurs, et en particulier "le goût commun de la terre, de la chasse, du manger et du boire" ( $T, 106)$ les relient étroitement. Thérèse voit clairement combien Bernard et son père se ressemblent. Ils ont tous deux de l'instruction, ils se distinguent des autres paysans : Bernard est "plus fin que la plupart des garçons" ( $T, 43)$ que Thérèse "eût pu épouser" $(T, 43)$ et son père est "[l]e seul homme supérieur qu'elle crût connâttre" (T, 103). Ils ont vécu ailleurs qu'à Argelouse et Saint-Clair: Bernard à Paris et le père Laroque à B. Ils sont tous deux pudibonds : le père s'empourpre aisément et Bernard quitte un music-hall "choqué" (T, 61). Au chapitre $X$, Bernard confirme à Thérèse que son père et lui sont "entièrement d'accord" (T, 153). Leur rôle de chef de famille et de notable propriétaire les modèle de façon similaire. Ainsi mesure-t-on à la fois la folie de 
Therèse et ses raisons lorsqu'elle choisit le mariage comme "un refuge" $(T, 53)$. C'est un second père qu'elle choisit, c'est-d̀-dire un second maitre - "A vous de les [quelques idées fausses] détruire, Bernard" ( $T, 55)$ - parce qu'elle pense qu'il la protègera. Mais tout comme son vrai père la rejette en refusant de l'accueillir après le non-licu- "Ah, ça non" $(T, 22)$ - Bemard l'abandonne à son désespoir.

La mère d'Anne peut apparaître comme une figure isolée de "la femme de la famille" jusqu'au chapitre VIII, mais à ce moment l'on comprend qu'Anne va évoluer jusqu'à lui ressembler tout à fait. Quand Marie révèle Anne comme une mère potentielle, la passion amoureuse perd de sa valeur face à cette dignité. Elle devient alors une "femme de la famille", dont le mariage avec Deguilhem et la future maternité sont le destin logique. En revanche, tout en ayant essaye de "s"incruste[r] dans un bloc familial" $(T, 53)$, "d"entr[er] dans un ordre" (T, 54), Thérèse n'a pas plus supporté sa vie que sa grand-mère Bellade; elle est génétiquement une renégade. Dans le chiasme esthétique fabriqué ici par Mauriac se lit un terrible déterminisme. Cependant ce donné génétique et culturel ne devient la seule réalité possible qu'avec le durcissement des coeurs et l'indifférence des personnages les uns pour les autres. De cette nouvelle dimension naissent d'autres liens entre les caractères.

Ainsi, si Anne finalement devient sa propre mère, elle est associable dans la première partie de l'ouvrage successivement à Jean puis à Therèse. En effet, dans les souvenirs de Therèse, au chapitre III, Anne adolescente est essentiellement pleine de vie, sans mesure, rêveuse, légère, cruelle, et ses paroles ne sont que jacasseries, comparable en cela à Jean qui sert à Thérèse un "ragoût" (T, 115) sans vérité, se révèle cruel avec Anne et vit dans la fantaisie, la gratuité et le jeu. Mais lorsque Anne tombe amourcuse de Jean, elle se dévêt de cet aspect puéril et par là même, cessant de lui ressembler, devient semblable à Thérèse du temps où cette dernière était amoureuse d'elle. En effet, tout comme Therèse la suivait à la chasse alors qu'elle "haïssait ce jeu" $(T, 48)$, Anne qui "haïssait la lecture" $(T, 46)$ envic maintenant sa culture à Thérèse . De même, elles abandonnent toutes deux l'initiative à l'être aimé - Therèse : "reviens quand le coeur t'en dira" (T, 49); Anne : "Nous nous arrêtons au bord de la dernière caresse (...) C'est lui qui me résiste" (T, 79-80) - et lui obéissent sans conditions. Elles ressentent la même torture en son absence, qui se traduit extérieurement par cet air hagard - Thérèse : "cette jeune fille un peu hagarde" $(T, 50)$; Anne : "aucune lumière dans les yeux" $(T, 92)$. Ces trois personnages presentent donc des points communs, mais en quelque sorte dans des temporalités différentes. De fait, ils demeurent incapables de communiquer. Ils se ressemblent à des années de distance, mais leur psychologie s'étant modifiee entre temps, il leur est impossible de se comprendre. Cette impossibilité de rejoindre à temps un être non déformé par la société, - et abandonné a l'expression non contrôlée de son 'génotype' - avant que ses rêves et sa vie se soient modifiés, me 
semble clairement exprimée dans ce passage du chapitre III (qui constitue également une annonce de leur destin) : "Des nuées orageuses leur proposaient de glissantes images; mais avant que Thérèse ait eu le temps de distinguer la femme ailée qu'Anne voyait dans le ciel, ce n'était déjà plus, disait la jeune fille, qu'une étrange bête étendue" ( $T, 47)$. Notons que le souvenir de la gravure "Cour d'Assises" que possède Bernard et celui de Thérèse à propos du criminel Daguerre (chapitre X) fonctionnent comme des révélateurs de la nature originelle commune de ces deux êtres, potentiellement capables de pitié pour autrui, mais trop durcis à l'âge adulte pour faire affleurer ces sentiments et les partager. Le narrateur suggère également au chapitre V qu'Hector de la Trave a connu la passion amoureuse dans sa jeunesse; il peut s'en rappeler la puissance mais ne peut entrer dans les vues d'Anne. Il paraît donc possible de distinguer deux types de ressemblance entre les personnages. Les premières se situent après que les individus ont renoncé à leur personnalité propre pour se mettre au service de la famille, entrer dans l'ordre. Telles sont les ressemblances entre Bernard et le père Laroque, Anne et sa mère, et celles que présentent les membres de la société. On peut les dire apparentes et définitives. Les secondes concement les personnages alors qu'ils ne sont pas encore soumis à l'idéologie familiale. Telles sont celles qui lient Anne à Jean puis à Therèse, mais aussi Anne à sa mère - lorsqu'elles sont amoureuses - et Bernard à Hector, quand leur souvenir secret leur permet d'éprouver de la compassion - Hector pour sa fille parce qu'il fut lui-même amoureux et Bernard pour Thérèse quand sur elle se superpose l'image "Cour d'Assises" au chapitre XV. Ce dernier type de ressemblance peut être dite secrète (non visible par autrui) et provisoire. Jusqu’à présent, le réseau de ressemblances entre les personnages et leurs oppositions semble finement tissé par Mauriac, mais une maille demeure plus lâche : Thérèse serait-elle isolée une fois mariée, sans aucun trait commun avec un autre personnage?

Il me semble que l'on peut établir un parallele entre le sort de Clara et celui de Thérèse. Nous avons déjà noté que Clara se trouve à la fois à l'intérieur et à l'extérieur de la famille, peut-être en est-il de même pour Thérèse. En effet, à ne considérer dans le livre que deux personnages, Thérèse et la famille, et la nature de leur conflit, on a souvent négligé l'appartenance de Thérèse à cette famille. Pourtant, elle en demeure un membre jusqu'à la fin de l'ouvrage, on ne l'a pas fait disparaître et elle en a accepté bien des conventions. Ainsi, l'on évoque souvent la solitude de Thérèse, mais on oublie de dire qu'elle a choisi, par exemple, de ne pas rencontrer le prêtre (du mystère duquel nous ne savons rien) parce que "cette démarche eût paru étrange à sa famille et aux gens du bourg" (T, 141). 5 Son attitude est-elle si différente de 'celle de Bernard lorsqu'il "accomplissait son devoir" ( $T, 146)$ en suivant la procession? Le qu'en dira-t-on guide leurs deux comportements.

Elle montre également "de la déférence” (T, 141) envers ses beaux-parents. 
Certes, cette double appartenance à soi et à la société ne constitue pas un élément suffisant pour établir une ressemblance entre Therèse et Clara. Mais la cristallisation sur la tante de nombreusès figures obsédantes du récit renforce peut- être le réseau d'assonances entre elles. Ainsi, l'un des thèmes récurrents du roman est le silence, par opposition au bruit qui évoque la vie. D'une manière plus spécifique, que nous révèle la parole ?

Les membres de la sociêté, et en particulier la famille, ne parlent jamais en lcurs termes propres. Ne cherchant pas à exprimer des idées personnelles, ils peuvent utiliser des formules conventionnelles, sans rechercher de formulation originale; ils parlent par dictons. "Tout est bien qui finit bien" $(T, 24)$ dit l'avocat au premier chapitre, "[o]n n'est jamais malheureux que par sa faute" (T, 42) est l'expression favorite de Bernard, “avec nous, ça ne prend pas” (T, 142) répète Madame de la Trave après son fils au chapitre VIII. “[C]omme à la prunclle de leurs yeux $(T, 86)$, "il ne faut pas se fier à l'apparence $(T, 121)$, les expressions toutes faites fourmillent dans leur parole. Au contraire, Therèse recherche l'expression qui correspondra exactement à ses pensees et à ses sentiments, notamment durant la laborieuse préparation de sa confession, puis à nouveau au chapitre XIII quand elle essaie d'expliquer son crime à Bernard. En revanche, lorsqu'elle se comporte, ou feint de se comporter, comme un membre de la famille, elle utilise à son tour un discours tout fait. "Donnez-vous les gants de céder un peu" ( $T, 84)$ dit-elle à sa belle-mère au moment où elle se ligue à la famille pour trahir Anne, puis elle accuse Jean "de porter le trouble et la division dans un interieur honorable" ( $T, 111)$. Ces formules constituent un signe d'appartenance auquel se fie la famille, et un voile sous lequel Therèse cache aisément ce qu'elle pense. alors que chez les autres, elles indiquent un renoncement à toute pensée personnelle. le bruit produit par leur discours n'est pas celui de la vie, mais plutôt de la mécanique dont ils constituent les rouages.

La parole de Clara se distingue de celle des autres membres de la famille et la rapproche, me scmble-t-il, de Thérèse. En effet, la tante, étant sourde, ne peut entendre la musique de la société, et ne peut par conséquent y adhérer ni de gré ni de force. L'on sait qu'elle ne parle sans cesse que pour ne pas avoir à essayer de comprendre. Son discours n'est qu'une longue accumulation de formules toutes faites, mais portées à un tel degré d’absurde qu'elles ne peuvent plus rien signifier, pas même les conceptions conventionnelles et étroites de la famille. Il est évident qu'elle n'accorde elle-même aucune signification à son propre discours. Ses paroles ne sont qu'un bruit émis pour se garantir de la mécanique. Comme Therèse, Clara se sert donc de ces formules toutes faites pour se protéger, mais sa pensée n'y est pas soumise. Ses radotages pourraient n'être qu'une preuve de gâtisme s'il n'était clair au chapitre IX qu'elle comprend, à sa manière intuitive, par son regard, le malheur de Thérèse et l'hypocrisie de Bcrnard. Elle sait donc pénétrer sous l'apparence et deviner la réalité. Cette caractéristique la différencie des autres membres de la famille car ils ont beau 
dévisager Thérèse, ils ne parviennent pas à connaître la vérité. La société restant impuissante devant l'art de la dissimulation de ses membres invente des raisons et des causes qui satisfont son bon sens sans rien expliquer. Ainsi, ne voyant pas Therèse pleurer après la mort de Clara, ils s'imaginent qu'elle l'a tuée. Clara, qui ne s'exprime qu'en caricaturant le language de la famille, cherche la vérité du monde qui l'entoure par son regard silencieux. Mais le silence est clairement associé à la mort par Therèse, ou du moins à cette non-vie d'Argelouse. Clara, par l'infurmité qui l'accable, rejoint Thérèse dans cette mort que constitue le silence. Sur un autre plan, Thérèse, comme Clara, est toujours réduite au 'silence' car son discours demeure déficient à ses propres yeux et à ceux de ses interlocuteurs qui ne la prennent pas au sérieux. Elle reste jusqu'au bout incapable d'exprimer totalement ce qu'elle pense et ce auquel elle aspire par des mots. Aphasique tout au long du roman, elle finira par communiquer un sentiment de compassion a Bernard par son corps seul. De même, lorsque Clara communiquera avec Thérèse - et le lecteur - cela s'effectuera par des actes, volontaires ou non, dans lesquels les mots n'auront qu'une fonction subsidiaire. Ces actes constitueront d'autres images qui rapprocheront de plus en plus ces deux personnages.

Ainsi, au chapitre X, Clara est dite "emmurée vivante" (T, 161) et bientôt Thérèse va se retrouver elle aussi enfermée physiquement dans la maison, comme elle avait êté prếcédemment emprisonnée par la famille, "cette cage aux barreaux innombrables et vivants" (T, 77). Liee a cette image de l'enfermement par ce qu'elle implique d'insuffisance, de manque et d'aspiration à plus, la figure de l'étouffement parcourt également tout l'ouvrage et confirme le lien qui unit Clara à Thérèse. 6

Therèse étouffe dans l'environnement naturel d'Argelouse, elle ćtouffe dans la sociêté, dans sa famille et dans son mariage. En réalité, la famille organise scrupuleusement l'asphyxie de ses membres qui ne survivent dans son sein qu'à condition de ne demander que peu d'oxygène. Pour elle, il s'agit d'étouffer l'affaire, d'étouffer le scandale, c'est-à-dire d'êtouffer celle qui l'a crế. Lorsqu'un membre du clan a transgressé ses lois, on le fait disparaître, on éli mine son existence, et Jean compare cette opération à un assassinat par noyade, où l'on retrouve implicitement l'image de l'étouffement. Bien que Thérèse suggère que ses ancêtres ont été tuberculeux ou syphilitiques et que Bernard souffre du coeur, la seule personne que nous voyions souffrir physiquement d'étouffement est Clara : "Tante Clara, soufflant, gravit l'escalier" (T, 161). Dans le même chapitre, l'asphyxie totale de Thérèse ayant été décidêe par la famille, celle-ci ne pouvant la supporter décide de se suicider. A ce moment, Clara prend en quelque sorte cet étouffement à son compte et meurt à la place de Thérèse. Mais la figure de l'étouffement ne s'épuise pas ici. Elle est renouvelée depuis le chapitre VIII et tout au long du chapitre IX par les scènes où Thérèse ouvre et ferme une fenêtre, pour s'échapper. Or les pins matérialisent aussitôt 
son inévitable emprisonnement, ils constituent le symbole de son impuissance. Ces pins représentent l'immobilisation de sa fortune, et de fait son inutilite, son bien s'est retourné contre elle, les pins deviennent "ses gardiens" (T, 171), une "armée ennemie" (T, 171). Ils sont l'image extériorisée ct agrandie des barreaux de la cage familiale. Ils sont la manifestation incontournable de l'art du traquenard et des retournements de situation de la famille; de l'impossibilité absolue de la fuir car elle tue, physiquement ou moralement. toujours indirectement. mais de façon certaine. Les pins, la fortune de la famille, deviennent ici son instrument de cocrcition, inévitables car "ils cernaient la maison" (T, 171), et inoubliables car "leur odeur résineuse emplissait la nuit" (T, 171). ${ }^{7}$ Au chapitre $X I$, les sensations liées aux pins seront finalement converties. De la fenêtre parviendra à Thérèse un "souffle glacê" $(T, 203)$ - évoquant peut-être le salon de Clara décrit par Thérèse comme une "glacière" ( $T, 45)$ - qui rompra l'étouffement. Elle, qui vient de rêver d' "un banc devant la mer" $(T, 200)$, peut comparer à présent "la rumeur des pins" (T, 203) à un "bruit d'Océan” (T, 203). c'est-à-dire à la liberté. Thérèse commence en effet à se libérer. Pour la première fois elle tâche d'amadouer sa souffrance, s'en fait un “jeu” (T, 203). En l'augmentant volontairement, elle la vainc. Enfin, elle va la convertir en béatitude. C'est donc en exposant son corps qu'elle affronte pour la première fois la pression familiale et s'en libère. A la regarder superficiellement, cette soudaine liberration de Therèse ressemble à un deus ex machina permettant à Mauriac de la faire sortir de cette chambre. En réalité, un retournement a commencé de s'accomplir dès le chapitre VIII, lorsque Thérèse, en même temps qu'elle devient criminelle, découvre à la fois Clara et la nature de la vraie liberté.

Jusqu'à ce point, d'une part Therèse ne remarque pas Clara - "Pas plus qu'un dicu ne regarde sa servante, je ne prêtais d'attention à cette vieille fille" $(T, 107)$ et plus loin “... moi qui ne la voyais même pas” $(T, 108)$ _ ; d'autre part, elle cherche l'image de la liberté et de l'indépendance d'esprit en des êtres qui la décevront. Elle n'admire et ne s'éprend que de la forme de liberté qui caractérise Jean et Anne avant sa passion. Or cette liberté faite essentiellement de caprice et de légèreté sera facilement brisée par la famille lorsqu'elle voudra se transformer chez Anne en affirmation de soi, et ne constitue que la soumission au code tout aussi vide d'un autre milieu chez Jean. Therèse n'aperçoit pas la liberte de la tante, scule capable d'agir gratuitement et par compassion, sans se compromettre avec la famille et sans en être totalement rejetée.

En réalité, le narrateur ne nous précise pas que Clara secourt les métayers par compassion - Thérèse prétend même qu'elle n'aime qu'elle -, simplement elle le fait comme si cela allait de soi. Mais nous savons qu'elle compatit avec Therèse lorsqu'au chapitre VIII l'on nous dit : "Thérèse faisait pleurer tante Clara en affirmant qu'elle finirait comme sa mère". Thérèse pleure aussi sincèrement, mais sur elle-même : au chapitre $V$, en songeant a la douce souffrance qu'apporte la passion, puis au chapitre $X$ devant son enfant et lorsqu'elle 
songe à se suicider. Les autres membres de la famille ne pleurent ni sur euxmêmes - puisqu'ils n'ont aucune conscience des limitations de leur vie - ni sur les autres - puisqu'ils n'éprouvent aucun sentiment de compassion.Clara, elle, n'a pas honte de sa pitié, en cela elle conserve la spontanéité et le naturel des enfants, elle illustre parfaitement l'image du simple en esprit des Béatitudes. Dans ce pays de sécheresse, où les êtres qui veulent s'accomplir intellectuellement et amoureusement sont dits consumés ("sa [Thérèse] face de brûlée vive" $(T, 27)$ - "leur enfant [Anne] consumée" $(T, 85)$...), Clara est celle qui donne "à boire" ( $T, 45)$, qui apaise. Ce rôle se trouve confirmé, me semble-t-il, dans le rapport à la nourriture que chaque personnage du roman manifeste. En effet, les membres de la famille aiment la viande : ils consomment le produit de leur chasse; la famille est une grande dévoreuse d'êtres vivants. Au contraire, Anne amourcuse se nourrit de fruits. Quant à Thérèse, depuis le moment où elle se maric, elle ne prend plus aucun plaisir à s'alimenter, bien évidemment parce qu'elle est enceinte, mais surtout parce qu'elle est avide de nourritures spirituelles, les aliments que la famille lui offre l'écoeurent. La démarche de Clara est alors de lui offrir des sucreries, c'est-à-dire des douceurs, de la tendresse. Clara est trop simple pour lui fournir les joies auxquelles elle aspire, mais elle tâche de lui faire plaisir et de la ramener au bonheur simple de l'enfance. Pourtant elle semble échouer sur le moment. Même les nourritures que Thérèse aimait parce qu'elles étaient associées à des moments de bonheur avec Anne, comme "un oeuf frit sur du jambon" ( $T, 32)$ finissent par la dégoûter lorsqu'elle se retrouve à Argelouse : "Balionte lui ayant monté un oeuf frit sur du jambon, elle refusa d'en manger; ce goût de graisse l'écoeurait à la fin ! “ (T, 196). Thérèse, claustrée, finit par ne plus manger du tout. Mais dans cette austéritemême s'accomplit le renversement. A la fin de l'ouvrage, Thérèse boit jusqu'à l'ivresse ("Un chaud contentement lui venait grâce à cette demi-bouteille de Pouilly" (T, 240)) et enfin : "Elle eut faim" ( $T, 239)$. Cependant, si Clara ne parvient pas à la nourrir, elle sait atténuer les chocs entre Thérèse et le monde.

Ainsi, au chapitre VIII lui fournit-elle un bouclier contre l'agressivité du père Laroque. De même, quand au chapitre IX "Tante Clara s'ass[o]it entre les deux époux" (T, 160), ses “jacassements” (T, 161) different la scène du salon. Elle semble également chercher par ses attentions à empêcher le conflit de se produire : "Vous ne vous couchez pas ? Thérèse doit être fourbue. Tu trouveras dans la chambre une tasse de bouillon, du poulet froid" $(T, 161)$. Le personnage de la tante constitue aussi un tampon entre Thérèse et le crime, puisqu'au chapitre VIII sa "crise aiguë de rhumatismes" $(T, 152)$ contraint Therèse à la relayer, ce qui semble - d'après les dates - retarder l'empoisonnement final, de l'époque de la chasse à la palombe jusqu'en décembre. Thérèse remarquera elle-même que Clara "s'est couché[e] sous ses pas au moment où elle allait se jeter dans la mort" $(T, 185) .8$ Il est à noter que Clara apaise les vrais conflits mais envenime les discussions idiotes (chapitre VI). En se mettant entre les dan- 
gers qui menacent Thérèse et celle-ci, Clara jusqu'à sa mort tente de l'en protéger. Mais ne déduisons pas de toutes ces remarques que Clara est parfaite, car aucun des personnages de Mauriac n'est monolithique. Nous apprenons en effet au chapitre X que Clara a chassé la palombe : "Cette pélerine délavée a une poche profonde : tante Clara y rangeait son tricot du temps qu'clle aussi, dans un 'jouquet' solitaire, guettait les palombes" ( $T, 180)$. Clara a donc elle aussi fait preuve de cruaute; parce qu'elle apparticnt à la famille, qu'elle est prise dans une hérédité et un milieu, elle ne peut cetre innocente ni du crime commis sur Thérèse - sa réduction intellectuelle, spirituelle et physique - ni du crimc que Therèse a commis: elle cst à la fois victime et coupable. Cette faille de cruauté chez. Clara vient en symétrie des failles sentimentales chez les autres. Aucun personnage de Thérèse Desqueyroux n'est ange ou bête. Pourtant, la chasse n’appartient plus qu'au passé de Clara et elle n’est plus, peu avant sa mort, que dévouement compatissant.

Ainsi, lorsqu'au chapitre XI, Thérèse arrive à Argelouse, Clara et Bernard l'accompagnent dans ce qui devient une sorte de montée au supplice. Dans les scènes suivantes, la tante ne cesse de monter et descendre les escaliers pour protéger Thérèse ("gravit l'escalier" (T, 161), "redescendit à tâtons" (T, 161), "gagna la piecce qu'elle occupait au-dessus du grand salon" (T, 175)). Bemard voudra la maintenir en haut, à l'écart, et l'obstination de Clara à essayer de faire monter Thérèse lui coûtera finalement la vie. Cependant, notons bien qu'elle meurt en haut, c'est-à-dire, à la fois dans une perspective chrétienne et dans l'optique du roman, sans doute sauvée. Car le thème de l'ascension et surtout de la chute parcourt l'ouvrage. 9

Contrairement à Clara qui demeure identique à elle-même tout au long de l'ouvrage, les autres membres de la famille, bien qu'ils puissent faire preuve de libertê, reviennent toujours aux conventions sociales : leur liberté, c'est-à-dire en termes familiaux leur faiblesse, est tôt ou tard auto-censurée. Au chapitre XII, sans faiblesse devant sa propre douleur, Therèse tâchera de fairc bonne figure pour ne pas effrayer Deguilhem, jouant ainsi le rôle que la famille attend d'elle, mais non sous la menace, librement - comme Clara se dévouait pour les métayers. Mais ce sens de la gratuité n'interviendra qu'à la fin du récit. Entre le moment où Thérèse devient criminelle et celui où elle atteint la grandeur d’âme de Clara, de quelles caractéristiques de la famille s'est-elle libérée et qu'a-t-elle hérité de la tante?

Jusqu'au chapitre XI, Thérèse attend son salut de l'extérieur, d'un artifice : de Jean, du prêtre, de la mort de Bernard, puis de son pardon lorsqu'elle prépare sa confession, de son suicide enfin. Elle a d'ailleurs toujours fui dans l'illusion, le rêve, l'espoir vague qu'elle n'aurait pas à construire sa destince, puis à l'assumer, qu'clle pourrait s'en remettre aux autres. Aussi s'est-elle mariée pour ne plus avoir à réflecchir. Dès le premier chapitre, Therèse fuit ses propres penscees dans l'observation du paysage, puis au chapitre II elle s'enfuit dans le rêve. 
Elle tente de fuir la confrontation avec Bernard en déclarant à l'avocat son désir de retourner chez son père. Plus loin, elle tâche d'éviter la première rencontre avec Jean, elle fuit son enfant et, lors de son retour, les gens de sa région. Elle n'assumait ses pensées qu'en paroles, encore demeuraient-elles sans impact. Cependant, sa tentative d'assassinat et la répression à laquelle la famille va la soumettre vont la contraindre à une solitude presque totale dans laquelle elle ne pourra plus échapper ni à un face-à-face avec elle-même, ni à une homologie de plus en plus frappante avec Clara.

En effet, dès le chapitre VIII, dans le même temps où elle laisse Bernard s'intoxiquer puis poursuit son empoisonnement, elle relaie Clara dans ses tâches charitables, puis fait des efforts pour calmer son inquiétude ("Elle avait trouvé La force de sourire à sa tante, de lui tenir la main" (T, 156)), à sa mort "Thérèse parle dans son coeur à celle qui n'est plus là" (T, 185), et la "regarde" (T, 185) enfin. Claustrée, elle demeure sourde aux paroles de Balionte au chapitre XI, et devient laide aussi. A ce point culminant de sa déchéance physique, elle se dépouille totalement de son orgueil et la rencontre avec Deguilhem peut avoir lieu. Son apparition, par sa coïcidence avec le souvenir de Bernard, va provoquer sa liberation. Mais peut- être une vision un peu semblable à celle de Genêt infiltre-t-elle le roman. Aux êtres auxquels n'ont pas été données de souffrances par Dieu, le salut ne serait-il possible que dans les souffrances impliquées par le crime? En clair, n'était-il pas nécessaire que Thérèse 'tuât' pour être rédemptée? Quoi qu'il en soit, à ce moment, l'apparence et la réalité se recouvrent exactement dans les actes de Thérèse, comme cela avait toujours été le cas pour ceux de Clara.

Au contraire, tous les actes de la famille répondent à un intérêt égoiste, c'est pourquoi elle n'est pas libre. Ainsi, Bernard suit la procession pour faire son devoir, c'est-à-dire en fait pour conserver sa bonne réputation; sa mère et lui sont attentifs à la santé de Thérèse lorsqu'elle est enceinte parce qu'elle porte l'héritier des pins; et Bernard et Laroque sauvent Thérèse de la prison pour l'honneur de la famille. Leurs objectifs primordiaux sont résumables en deux points principaux : accumuler de la terre et maintenir une bonne réputation, car seule l'apparence de la morale leur est nécessaire. En fait, la famille est amorale. Toutes les stratégies lui sont bonnes pour conserver la position éminente de ses membres et développer son capital foncier par une suite d'unions profitables. Ainsi le chantage est un moyen largement utilisé : par Laroque au début du chapitre premier "Dieu merci, on tient le directeur de La Lande Conservatrice" ( $T, 19)$, par Madame de la Trave au chapitre V (“D'ailleurs, nous la tenons" ( $T, 85)$ ) et par Bernard au chapitre IX ("moi, je vous tiens" ( $T$, 165)). Notons que la similarité de leurs méthodes s'alourdit du fait qu'ils usent de la même expression. Le mensonge, la menace, le chantage permettent d'entretenir une façade que le reste de la société ne peut percer. Il apparaît alors que la famille s'évalue elle-même non selon la réalité - qu'elle connait mais ne 
perçoit pas dans sa laideur - mais selon l'effet de son apparence sur les autres. Elle se juge dans le miroir déformant de sa réputation en évitant de se regarder elle-même. Elle prévient ainsi les conditions de toute libération.

Thérèse ment aussi à tout le monde et pour son propre compte jusqu'au chapitre XII. Elle ment à Anne, lorsqu'elle prétend servir son amour alors qu'elle ne conspire qu'à sa destruction; à la famille, quand elle parait en servir les interêts en tâchant d'influencer Anne; à Bernard qu'elle empoisonne en semblant le soigner. Chez elle aussi, l'apparence est trompeuse et ses actes assouvissent, sinon ses intérêts, du moins sa rancune et son besoin de vengeance : malheureuse, elle ne désire pas voir le bonheur fleurir autour d'elle. Elle use également de la menace, si elle se sent en danger. Ainsi au chapitre IX. $s$ adressant à Bernard, elle dit : "Vous ne pouvez plus vous déjuger. Vous seriez convaincu de faux témoignage ..." $(T, 165)$ et "Croyez-vous donc que vous me retiendrez de force" (T, 172). Cependant, tout en recherchant confusément l"issue de sa prison, elle se sait coupable. Elle reconnaît l'horreur de certains de ses gestes puisqu' elle a du mal à croire qu'elle ait pu les accomplir (chapitre IV : "J'ai fait cela. C"est moi qui ai fait cela ..." (T, 71)). Tout au long du livre, elle explore les raisons de ses actes, tâche de retrouver les conditions dans lesquelles ils ont eu lieu et leurs motivations. Mais jamais elle ne se justifie de ses crimes. sauf dans la colère (chapitre IX : "Cela n'aurait eu aucune importance que cet imbécile disparût du nombre des vivants" (T, 164-165)). D'ailleurs, si Thérèse en rentrant à Argelouse se sent protégée par la nuit qui lui permet de se cacher. elle n'aspire en fait qu'à la lumière: "ne rien laisser dans l'ombre" $(T, 29)$ serait son idéal. Toutes les images de bonheur - associées chez elle à des êtres jeunes se situent dans la lumière : courses folles avec Anne, promenades avec Jean. La famille par contre abrite sa réputation dans l'ombre de ses salons, et Bernard dit à Thérèse au chapitre V : "N'allume pas à cause des moustiques" (T, 96). pour finalement la laisser "dans le noir" ( $T, 174)$ au chapitre IX là où son père l' avait conduite "au crépuscule" ( $T, 14)$. Cependant, son besoin de clarté et la conscience de sa culpabilité, puisqu' elle espère être "pardonnée" $(T, 33)$ au chapitre II, ne signifient nullement qu'elle éprouve du repentir - peut-être en raison du caractère nécessaire de ce crime et de la nécessaire culpabilité en résultant. Elle élimine cette étape entre la confession qu'elle prépare au début du livre et son rêve d'absolution. C'est sans doute pourquoi la "confession" $(T, 29)$ devient une "défense" (T, 38) dès le chapitre II. Thérèse ne possède pas plus de scrupules que le reste de la famille dans les méthodes qu'elle utilise pour parvenir à ses fins. Mais à la différence de la famille, ce n'est pas sur l'apparence qu elle a longtemps donnée qu'elle se juge. Au contraire, elle cherche la vérité de son être avec obstination au milieu de la logique folle de ce monde qui détruit les êtres. Cependant, sa démarche demeurant purement intellectuelle ne peut la conduire à se mettre à la place des autres. Elle les regarde, à distance, sans compassion : au chapitre V elle sent "souffrir dans l'ombre" $(T, 94)$ Anne "mais 
sans aucune pitié" $(T, 94)$, au chapitre VI elle inquiète Bernard sur son état par pure cruauté, puis ne paraît pas compatir aux souffrances qu'il ressent sous l'effet du poison. Seul l'accomplissement du destin de Clara ouvrira son coeur à la compassion et lui permettra de vivre la catharsis du chapitre XII et d'en sortir libre. Cependant, pour boire il ne fallait plus être "en nage" ( $T, 45)$, comme avait dit Clara : pour que la catharsis s'accomplisse, il fallait que Therèse, affrontant sa souffrance, la maîtrise et s'appaise. La porte qui s'ouvre au début du livre sur la liberté [chapitre I : "L'avocat ouvrit une porte" (T, 11)] - et se referme lors du mariage [chapitre IV : "au fracas de la lourde porte refermée" (T, 57)] - s'ouvre finalement bien sur la liberté [chapitre XII: "la porte que Thérèse ouvre enfin" (T, 211)].

Au cours de cette étude, Clara s'est d'abord révélée un guide éclairant au sein du fin maillage qui relie les personnages dans un réseau de ressemblances et d'oppositions subtiles dont le dessin au cours du récit se modifie et se nuance jusqu'à provoquer des retoumements inattendus. Elle a également joué le rôle d'un catalyseur d'images qui ont constitué le contrepoint structuré de l'intrigue du roman. En la suivant, il a été possible d'explorer les assonances et les dissonances des personnages, les rythmes marqués par la répétition des figures, les relations de certaines isotopies. Bien évidemment, elle ne permet pas d'épuiser le jeu des annonces et des symboles qui innervent cette oeuvre autant romanesque que poétique. Mais elle nous a permis d'envisager une réponse possible au mystère de l'amour de Mauriac pour son monstre Thérèse, si du moins il est vrai qu'il l'a rendue coupable au sein d'une société oublieuse de son enfance, afin de la sauver.

Purdue University 


\section{Notes}

1 Je me situe ici dans l'approche définie par J. E. Flower dans son article "Tunnels in Thérèse Desqueyroux.", paru dans Australian Journal of French Studies - May-Aug 1985 - à la page 127 : "an approach which is consemed less with Therèse individually as a character, than with the novel as a fictional construct in which various stylistic and structural elements are interdependent."

2 Toutes les citations, référées $(T, n)$, sont extraites de l'édition Grasset de 1927 de Thérèse Desqueyroux de François Mauriac.

${ }^{3}$ Comme le fait remarquer J. E. Flower dans l'article précedemment cite: "We see that she has always shown some inclination at times to accept her lot and to prefer security, however contradictory, given her actions, this may appear." (131)

4 Martin Turnell dans son article "The Style of François Mauriac" publie dans le volume 164 de Twentieth Century (1958), développe fort bien le sens et l'importance de cette figure dans le roman :

In Thérèse Desqueyroux, the most opressive of the novels.Mauriac makes extraordinary play with the word 'étouffer' and its variants. They reverberate all through the book with a soft thud like the muffled sound of footsteps falling on sand, or on a bed of pine needles. All the principal characters use them, but they arouse different emotions and different associations in different minds. They mean to suppress a scandal; they also mean to suppress or blot out the person who caused the scandal, as Therese will be suppressed. They describe the physical sense of suffocation of a hot day or a wet night, but they also describe the sense of psychological constriction or frustration which seems to result from physical conditions. In this way, they become the expression of the conflict which separates Therèse from her family, but they are gradually woven into the psychological web in which she is trapped.

Je n'en reprends ici l'étude que dans l'optique d'une comparaison entre Clara et Therèse afin de montrer comment des points communs de plus en plus nombreux apparaissent entre elles.

5 Bernard Chochon a fort bien exprime cette idée dans "Signes et Figures dans Thérèse Desqueyroux" publié dans le volume 707 de La Revue des Lettres Modernes (1984) : “Sombre prison qu'Argelouse, prison morale qui n'a d'égal que son double : la prison physique de son paysage, d’un cadre qui étouffe sa victime". (21)

6 Alastair B. Duncan, dans son article "Despair and Hope in Thérèse Desqueyroux" publie dans le volume 54 de Modern Languages (1982). développe une interprétation du personnage de Clara qui me semble fort convaincante et dans le sens de laquelle j'abonde: "Her death is an expression of love. a sacrifice offered freely, albeit unconsciously, so that Therese may live. And 
behind that sacrifice, Mauriac is hinting at the sacrifice of Christ who is similarly, in the Christian view, a rejected and suffering servant offering Himself in love for others. Therèse has been given a sign." (171)

7 La figure de la chûte atténuée en descente et de l'élévation est encore une de celles qui lient Thérèse et Clara. La libération de Thérèse après le non-lieu s'amorce par la descente des escaliers du Palais de Justice, or Thérèse commence ici à purger une terrible peine. Son cauchemar dans la calecche qui constitue une preuve de sa culpabilité a lieu dans "la descente du ruisseau" (T, 28). Lorsqu'elle envisage le suicide pour la première fois au chapitre IV, elle songe à se jeter par la fenêtre, et la seconde elle doit redescendre du grenier dans sa chambre (chapitre X). Les occurences sont encore nombreuses de ces 'pentes' qui signifient toujours la déchéance, sa tentation ou sa révélation : au chapitre IV, Thérèse se souvient de son premier crime, passionnel, lorsqu'elle transperce la photo de Jean, juste au moment où le train "dans une descente se précipite" (T, 71). Jean, qui matérialise les tentations de Thêrèse, lui dit que "[1]es êtres comme [eux] suivent toujours des courants, obeissent à des pentes ..." (T, 118). Toutes ces dénivellations ponctuent le roman, comme des accords plaqués sur la mélodie qu'ils renforcent.

${ }^{8}$ En fail, dans le cauchemar du chapitre II s'amorce déjà cette identification. En effet, le juge parlait à Thérèse de "[c]ette vieille pélerine - celle dont vous n'usez plus qu'en octobre" (T, 28), or Therèse elle-même affirme au chapitre X gue la pélerine appartient à Clara.

9 Notons qu'elle s'oppose ainsi à Balionte qui en constitue la parodie. En effet, les services de Balionte sont rémunérés, elle pleure de compassion pour un cochon mais demeure insensible à la souffrance de Thérèse, et son essoufflement lorsqu'elle s'affaire dans la chambre de Thérèse témoigne de sa mauvaise volonté et non de son dévouement. La présentation du contraste entre les deux femmes semble constituer une nouvelle parabole du bon serviteur. 
Ouvrages cites

Bessan, Edma. "Solitude in the Novels of François

Mauriac." French Review 8 (1935) ; 129-134.

Brosman, Catherine S. "Point of View and Christian Viewpoint in

ThérèseDesqueyroux." Essays in French Literature 2 (1974): 69-73. Chochon, Bernard. "Le diable et le Bon Dieu : Sartre et Mauriac."

La Revue des Lettres Modernes 582-587 (1980) : 97-111.

- - ". "Signes et figures dans Thérèse Desqueyroux."

La Revue des Lettres Modernes 707-709 (1984) : 11-33.

Crisafulli, Alessandro S. "The Theme of Captivity and its

Metaphorical Expression in Mauriac's Thérèse Desqueyroux."

Studies in the Honor of Tatiana Fotitch. Eds Joseph M.

Sola-Sole, Alessandro S. Crisafulli and Siegfried A. Schultz.

Washington D.C. : Catholic U. of America P., 1973. 29-35.

Duncan A.B. "Despair and Hope in Thérèse Desqueyroux."

Modern Languages 54 (1982) : 168-172.

Farrel, C. Frederick Jr and Edith R.Farrel. "The Multiple Murders of

Therèse Desqueyroux." Hartford Studies in Literature 2 (1970) :

195-206.

- -. "Thérèse Desqueyroux : A Complete Suicide." Language Quaterly

14 (1976) : 13-15, 18, 22.

Fischler, Alexander. "Thematic Keys in François Mauriac's

Thérèse Desqueyroux and Le Noeud de Vipères." Modern

Language Quaterly 40 (1979) : 376-389.

Flower, J.E. "Tunnels in Thérèse Desqueyroux." Australian Journal

of French Studies May-Aug 1985 : 126-137.

Gallant, Clifford J. "La mère dans l'oeuvre de François

Mauriac." Kentucky Foreign LanguageQuaterly 11 (1964) : 79-85.

Genz, Réjane P. "Le rôle de la société dans l'affaire Thérèse

Desqueyroux." Kentucky Romance Quaterly 21 (1974) : 419-427.

Glénisson, Emile. L'amour dans les romans de François Mauriac.

Paris : Editions Universitaires, 1970.

Graef, Hilda. "Marriage and our Catholic Novelists." Catholic World 189 (1959) : 185-190.

Joubert, André J. François Mauriac et Thérèse Desqueyroux. Paris :

Nizet, 1983.

Kushner, Eva. François Mauriac. Paris : Desclée de Brouwer, 1972. Le Hyr, Yves. "François Mauriac : Thérèse Desqueyroux."

Etudes Classiques 39 (19) : 29-38.

Madaule, Jacques. "La Grâce dans l'oeuvre de François Mauriac." 
RLM 516-522 (1977) : 77-91.

Mauriac François. Thérèse Desqueyroux. Paris : Grasset, 1927. Mein, Margaret. "François Mauriac and Jansenism." Modern

Language Review (1963) : 516-523.

Moloney, Michael. "François Mauriac : The Way of Pascal." Thought 32 (1957) : 398-408.

Montférier, Jacques. "Etat présent des études sur François

Mauriac." L'Information Littéraire : Revue Paraissant Cinq Fois

Par An 35 (1983) : 162-165.

Montférier, Jacques. "Thérèse Desqueyroux, Phèdre et le destin." RLM 516-522 (1977) : 109-118.

Moraud, Aline. "La Femme, lieu du conflit dans les romans de

François Mauriac." RLM 707-709 (1984) : 35-52.

Mounin, Georges. "Structure, fonction, pertinence : A propos de

Thérèse Desqueyroux." Linguistique 10, i (1974) : 21-32.

Paschal, Mary. "Disappearance in the Novels of François Mauriac."

South Atlantic Bulletin 36 (1971) : 35-41.

Perrin-Naffakh, Anne-Marie. "Du bourgeonnement du texte à

l'affermissement du style." TCERFM 11 Juin 1982 : 7-22.

Quoniam, Theodore. "L'amour de Dieu." Magazine Littéraire Fév. $1985: 49-51$.

-. François Mauriac du péché à la rédemption. Paris : Téqui, 1984.

Séailles, André. "Le thème du pharisien et du publicain dans les romans de François Mauriac." L'information Littéraire 24 (1972) : 134-138.

Sjursen, Nina. "Thérèse Desqueyroux, une Phèdre moderne ? "

Orbis Litterarum 40 (1985) : 348-356.

Sutton, Geneviève. "Phèdre et Thérèse Desqueyroux : Une

communauté du destin." French Review 43 (1970) : 559-570.

Swift, Bernard C. "Structure and Meaning in Thérèse

Desqueyroux." Wascana Review 5 (1970) : 33-44.

Turnell, Martin. "The Style of François Mauriac: Sin and the

Novelist." Twentieth Century 164 (1958) : 242-253.

Vidrine, Donald R. "Thérèse Desqueyroux : Archetypal

Wanderer." Publications of Arkansas Philological Association

Fall 1982: 77-85.

Villas, James M. "The Pines Trees in Mauriac's Thérèse

Desqueyroux." Romance Notes 3 (1962) : 3-9.

Wildgen, K. "Dieu et Maman : Women in the Novels of

François Mauriac." Renascence 27 (1974) : 15-22. 




\section{Trois études sur un passage du Rouge et le noir}

The three following articles treat various aspects of a passage in Stendhal's Le Rouge ct le noir as transcribed below.

1 Cette magnificence mélancolique, dégradée par la vue des briques nues et

2 du plâtre encore tout blanc, toucha Julien. Il s'arrêta en silence. A l'autre

3 extrémité de la salle, près de l'unique fenêtre par laquelle le jour pénétrait

4 il vit un miroir mobile en acajou. Un jeune homme, en robe violette et en

5 surplis de dentelle, mais la tête nue, était arrêté à trois pas de la glace. Ce

6 meuble semblait étrange en un tel lieu, et, sans doute, y avait été apporté de

7 la ville. Julien trouva que le jeune homme avait l'air irrité; de la main

8 droite il donnait gravement des bénédictions du côté du miroir.

9 Que peut signifier ceci? pensa-t-il. Est-ce une cérémonie préparatoire

10 qu'accomplit ce jeune prêtre? C'est peut-être le secrétaire de l'éveque...il

11 sera insolent comme les laquais..ma foi, n'importe, essayons.

12 Il avança et parcourut assez lentement la longueur de la salle, toujours

13 la vuc fixcée vers l'unique fenĉtre et regardant ce jene bomme que

14 continuait à donner des bénédictions exécutées lentement mais en nombre

15 infini, et sans se reposer un instant.

16 A mesure qu'il approchait, il distinguait mieux son air fâché. La richesse

17 du surplis garni de dentelle arrêta involontairement Julien à quelques pas du

18 magnifique miroir.

19 Il est de mon devoir de parler, se dit-il enfin; mais la heauté de la salle

20 l'avait ému, et il était froissé d'avance des mots durs qu' on allait lui

21 adresser.

22 Le jeune homme le vit dans la psyché, se retourna, et quittant subitement

23 l'air fâché, lui dit du ton le plus doux:

24 -Eh bien! Monsieur, est-elle enfin arrangée?

25 Julien resta stupéfait. Comme ce jeune homme se toumait vers lui,

26 Julien vit la croix pectorale sur sa poitrine: c'était l'évêque d'Agde. Si

27 jeune, pensa Julien; tout au plus six ou huit ans de plus que moi!...

28 Et il eut homte de ses éperons.

Stendhal, Le Rouge et le noir. Paris: Gallimard (Pléiade), 1952, 314-315. 
